\title{
Dexmedetomidine alleviated sepsis-induced myocardial ferroptosis and septic heart injury
}

\author{
CHUNYAN WANG ${ }^{1}$, WENLIN YUAN $^{1}$, ANMIN HU $^{1}$, JUAN LIN $^{1}$, ZHENGYUAN XIA $^{2}$, \\ CATHERINE F. YANG ${ }^{3}$, YALAN $\mathrm{LI}^{4}$ and ZHONGJUN ZHANG ${ }^{1}$
}

\begin{abstract}
${ }^{1}$ Department of Anesthesiology, Shenzhen People's Hospital and Shenzhen Anesthesiology Engineering Center, The Second Clinical Medical College of Jinan University, Shenzhen, Guangdong 518000; ${ }^{2}$ Department of Anesthesiology,

The University of Hong Kong, Hong Kong, SAR, P.R. China; ${ }^{3}$ Department of Basic Sciences, College of Medicine, CA Northstate University, Elk Grove, CA 60009, USA; ${ }^{4}$ Department of Anesthesiology, First Affiliated Hospital of Jinan University, Guangzhou, Guangdong 510000, P.R. China
\end{abstract}

Received August 5, 2019; Accepted March 17, 2020

DOI: $10.3892 / \mathrm{mmr} .2020 .11114$

\begin{abstract}
Cardiac dysfunction resulting from sepsis may cause significant morbidity and mortality, and ferroptosis plays a role in this pathology. Dexmedetomidine (Dex), a $\alpha 2$-adrenergic receptor ( $\alpha 2-\mathrm{AR})$ agonist exerts cardioprotective effects against septic heart dysfunction, but the exact mechanism is unknown. In the present study, sepsis was induced by cecal ligation and puncture (CLP) in male C57BL/6 mice. Dex and yohimbine hydrochloride ( $\mathrm{YOH})$, an $\alpha 2-\mathrm{AR}$ inhibitor, were administered before inducing CLP. Then, $24 \mathrm{~h}$ after CLP, serum and heart tissue were collected to detect changes of troponin-I (TN-I), interleukin 6 (IL-6), superoxide dismutase (SOD), malonaldehyde (MDA) and glutathione (GSH) levels, and iron release. Ferroptosis-targeting proteins, apoptosis and inflammatory factors were assessed by western blotting or ELISA. It was found that, $24 \mathrm{~h}$ after CLP, TN-I, a biomarker of myocardial injury, was significantly increased compared with the control group. Furthermore, the levels of MDA, 8-hydroxy-2'-deoxyguanosine and the inflammatory factors IL-6 and monocyte chemoattractant protein-1 were also significantly increased. It was demonstrated that treatment with Dex reverted or attenuated these changes (CLP + Dex vs. CLP; $\mathrm{P}<0.05)$, but these protective effects of Dex were reversed by $\mathrm{YOH}$. Moreover,
\end{abstract}

Correspondence to: Dr Yalan Li, Department of Anesthesiology, First Affiliated Hospital of Jinan University, 613 West of Huangpu Road, Tianhe, Guangzhou, Guangdong 510000, P.R. China E-mail: tyalan@jnu.edu.cn

Dr Zhongjun Zhang, Department of Anesthesiology, Shenzhen People's Hospital and Shenzhen Anesthesiology Engineering Center, The Second Clinical Medical College of Jinan University, 1017 Dongmen Bei Road, Luohu, Shenzhen, Guangdong 518000, P.R. China

E-mail: luckydoczhang@163.com

Key words: glutathione peroxidase 4, heme oxygenase-1, ferroptosis, sepsis, heart
CLP significantly decreased the protein expression levels of glutathione peroxidase 4 (GPX4), SOD and GSH. However, CLP increased expression levels of heme oxygenase-1 (HO-1), transferrin receptor, cleaved caspase 3 , inducible nitric oxide synthase and gasdermin $\mathrm{D}$, and iron concentrations. It was found that Dex reversed these changes, but $\mathrm{YOH}$ abrogated the protective effects of Dex (CLP + Dex + YOH vs. CLP + Dex; $\mathrm{P}<0.05)$. Therefore, the present results suggested that the attenuation of sepsis-induced HO-1 overexpression and iron concentration, and the reduction of ferroptosis via enhancing GPX4, may be the major mechanisms via which Dex alleviates sepsis-induced myocardial cellular injury.

\section{Introduction}

Sepsis, which has a worldwide mortality rate of $10 \%$, is induced by infection and leads to life-threatening organ dysfunction (1). Clinical and basic research have shown that the cardiovascular system is affected during sepsis (2), but the exact mechanism is not fully understood. Dexmedetomidine (Dex), which has been recommended for patients with sepsis since 1999 , has been proven to be a selective $\alpha 2$-adrenergic receptor $(\alpha 2$-AR) $(3,4)$. Clinical studies have revealed that Dex inhibits the activity of sympathetic nerves via analgesic, sedative and antisialogogue effects $(5,6)$, while it also exerts protective effects on other organs, such as the kidney, brain and heart (7). Inflammation is the primary characteristic of sepsis; the expression of inflammatory factors, such as tumor necrosis factor $\alpha$ (TNF- $\alpha$ ), interleukin 1 (IL-1), IL-6 and methyl-accepting chemotaxis protein-1, are significantly increased during sepsis, and Dex is able to decrease these expression levels (8).

Ferroptosis was first reported by Dolma et al (9) in cancer cells and was shown to be different from the known cell death pathways, apoptosis, pyroptosis and necroptosis. Ferroptosis has since been identified to be involved in various pathological processes, including neurotoxicity, acute kidney failure, liver injury and heart disease (10), as well as myocardial ischemia reperfusion injury $(11,12)$. Furthermore, the development of sepsis has been proposed to involve ferroptosis (13). However, 
the role of ferroptosis in septic heart injury remains unknown. The mechanism of ferroptosis mainly involves increases in lipid peroxidation and further release of lipid reactive oxygen species (ROS) (14). It has also been reported that ferroptosis occurs when the activity of glutathione peroxidase 4 (GPX4) or glutathione (GSH) decreases (13). In addition, iron chelation has also been shown to inhibit ferroptosis, thus indicating that ferroptosis is closely associated with ROS and iron $(15,16)$. Furthermore, other factors, such as voltage-dependent anion channel 2 , heat shock protein $\beta$-1, nuclear factor E2-related factor 2 (Nrf2), NADPH oxidase, P53 and heme oxygenase-1 (HO-1), also participate in ferroptosis $(17,18)$.

The HO system, which includes HO-1 and HO-2, acts as a defense system against various stimuli, such as oxidants and hypoxia (19). Moreover, HO-1 degrades heme into carbon monoxide, biliverdin and ferrous iron, and confers cardioprotection via antiapoptotic, antioxidant and other effects. $\mathrm{HO}$ is one of the intracellular sources of iron (20), and its overexpression and activation have been shown to accelerate ferroptotic cell death (21). Furthermore, HO-1 participates in ferroptosis via its association with iron and its antioxidant effects (22), but the exact mechanism remains unknown. As previously reported, Dex reduces $\mathrm{H}_{2} \mathrm{O}_{2}$-induced oxidative stress in neonatal rat cardiomyocytes by decreasing ROS and GSH (23). According to a previous study (22), it is hypothesized that HO-1-mediated regulation of ferroptosis may play a role during sepsis and that Dex confers cardioprotective effects by influencing this regulation.

\section{Materials and methods}

Cecal ligation and puncture (CLP) operation. A total of 32 male C57BL/6 mice ( $25 \mathrm{~g}$, 8 weeks old) were obtained from the Guangdong Medical Lab Animal Center and housed in the Laboratory Animal Service Center (Jinan University, Guangdong, China). Mice received standard care under a 12-h dark/light cycle $\left(23^{\circ} \mathrm{C}\right.$ with an atmosphere of $\left.60 \%\right)$ and were given free access to food and water, in accordance with the Animal Care guidelines of the Jinan University. The study was approved by the Institutional Ethics Committee of The Medical Committee of Shenzhen People's Hospital (approval ID: LL-KY-2019604). Sepsis was induced by CLP, as previously described (24). Briefly, mice were anesthetized with isoflurane (RWD Life Science) inhalation at the concentration of $2.5 \%$ for anesthetic induction and then at $1 \%$ for anesthetic maintenance until the end of the CLP. During the experiment, the body temperature was kept at $36-38^{\circ} \mathrm{C}$ with a heating pad. Anesthetized mice were subjected to midline laparotomy. The cecum was carefully separated to avoid blood vessels damage and the cecum was identified and punctured twice with a 22-gauge needle. Then, the abdominal cavity was closed with two epithelium layers, followed by a normal saline injection subcutaneously for resuscitation before mice were returned to the cage. The duration of the whole experiment was $\sim 25 \mathrm{~h}$ and the CLP model was finished within $15 \mathrm{~min}$. The cecum was exposed immediately following laparotomy, which was conducted using a $2-\mathrm{cm}$ lower midline incision, followed by the use of a 22-gauge needle to impale the cecum. Before the cecum was returned to the abdominal cavity, pieces of feces were extruded. The abdomen was closed with 4-0 silk sutures in two layers. The health and behavior of the animal was monitored at $1 \mathrm{~h}$ intervals over $12 \mathrm{~h}$. During the experiment, the survival rate (rate of successful CLP model establishment) was $60 \%$ in the CLP group, $86 \%$ in CLP + Dex group and $70 \%$ in CLP + Dex + yohimbine hydrochloride (YOH) group. It was found that the primary reason for mortality was the individual variation in the reaction to CLP-induced sepsis. To ensure a minimum of six live animals per group for the experimental period, ten, seven and nine mice were initially used in the CLP, CLP + Dex and CLP + Dex + YOH groups, respectively. At the end of the experiments, an overdose of phenobarbital sodium was used for euthanasia and then blood $(500 \mathrm{ml})$ and heart samples were collected.

Animal experimental protocol. The mice were randomly divided into 4 groups ( $\mathrm{n}=6$ per group): Control (Ctrl), CLP, CLP + Dex and CLP + Dex + YOH groups. Dex was administered $15 \mathrm{~min}$ before inducing sepsis at a dose $50 \mu \mathrm{g} / \mathrm{kg}$ and $1 \mathrm{mg} / \mathrm{kg} \mathrm{YOH}$ was given $30 \mathrm{~min}$ before the administration of Dex, according to our preliminary experiments and previous reports (25-27). Survival was $90 \%$ in the Dex administration group. A total of $500 \mathrm{ml}$ of blood was collected to separate serum and the hearts were harvested after $24 \mathrm{~h}$ of sepsis. Dex was obtained from Jiangsu Hengrui Medicine Co., Ltd., and YOH from MedChem Express.

Determination of troponin-I (TN-I), IL-6, methyl-accepting chemotaxis protein 1 (MCP-1), malonaldehyde (MDA), superoxide dismutase (28), 8-hydroxy-2'-deoxyguanosine (8-OHDG) and GSH expression. After $24 \mathrm{~h}$ of CLP, blood samples were collected and then centrifuged $(5,000 \mathrm{~g}$, $10 \mathrm{~min}, 4^{\circ} \mathrm{C}$ ) to separate the serum for the detection of TN-I (cat. no. JL31923), MDA (cat. no. JL13329), SOD (cat. no. JL12237), 8-OHDG (cat. no. JL12294), GSH (cat. no. JL20360), IL-6 (cat. no. JL20268) and MCP-1 (cat. no. JL20304). These ELISA kits were obtained from Shanghai Jianglai Biological Technology Co., Ltd., and used following the manufacturer's instructions.

Iron concentration detection. To detect iron concentration in the serum during sepsis, an iron assay kit (cat. no. ab83366; Abcam) was used according to the manufacturer's protocol.

Hematoxylin and eosin $(H \& E)$ staining. Briefly, the heart was fixed with $4 \%$ paraformaldehyde overnight at room temperature, embedded in paraffin and sliced into $4 \mu \mathrm{m}$-thick slices. The tissues were stained with hematoxylin for $5 \mathrm{~min}$ and eosin for $1 \mathrm{~min}$ at room temperature using the H\&E Staining kit (cat. no. C0105; Beyotime Institute of Biotechnology). The slide was observed under a fluorescence microscope (DMi8 DFC7000 T; Leica Microsystems, Inc.).

Western blot analysis. Frozen ventricular tissue samples were homogenized in RIPA buffer (Cell Signaling Technology, Inc.; cat. no. 9806) and centrifuged at $4^{\circ} \mathrm{C}, 13,200 \mathrm{x}$ g for $30 \mathrm{~min}$. The supernatant was collected for total protein analysis and the protein concentration was determined using a Bradford protein assay. Equal amounts of protein $(30 \mu \mathrm{g})$ from mouse heart homogenate were resolved by 7.5-12.5\% SDS-PAGE and subsequently transferred to PVDF membranes. The 
membranes were then blocked in TBS with $0.1 \%$ Tween-20 (TBST; cat. no. P9416; Sigma-Aldrich; Merck KGaA) containing $5 \%(\mathrm{w} / \mathrm{v})$ non-fat milk for $1 \mathrm{~h}$ at room temperature, after which the membranes were incubated with primary antibodies overnight at $4^{\circ} \mathrm{C}$. Primary antibodies against Bax (cat. no. 2772; Cell Signaling Technology, Inc.), Bcl-2 (cat. no. 210774; Merck KGaA), HO-1 (cat. no. ab13248; Abcam), GPX4 (cat. no. PAS79321; Thermo Fisher Scientific, Inc.), gasdermin D (GSDMD; cat. no. ab219800; Abcam), nitric oxide synthase (iNOS; cat. no. ab3523; Abcam), cleaved caspase 3 (cat. no. 836; Cell Signaling Technology, Inc.), P53 (cat. no. NB200-103; Novus Biologicals; Bio-Techne Ltd.), transferrin receptor (TFR; cat. no. MCA155R; Bio-Rad Laboratories, Inc.) ferritin (cat. no. ab75973; Abcam), Caspase 9 (cat. no. ab184786; Abcam), Nrf2 (cat. no. ab31163; Abcam) and GAPDH (cat. no. 5174; Cell Signaling Technology, Inc.) were used at the dilution 1:1,000. After washing with TBST three times for $10 \mathrm{~min}$ per wash, the membrane strips were incubated at room temperature with a 1:10,000 dilution of an anti-rabbit IgG (cat. no. 7074S; Cell Signaling Technology, Inc.) or anti-mouse IgG (cat. no. 7076S; Cell Signaling Technology, Inc.) secondary antibody conjugated to horseradish peroxidase for $1 \mathrm{~h}$. Protein bands were detected by chemiluminescence (cat. no. P90720; EMD Millipore) and the images were quantified using ImageJ version 1.51 software (National Institutes of Health).

Statistical analysis. Data are presented as the mean \pm SEM. All data were normally distributed, as confirmed by the GraphPad Prism normality test. Differences among multiple groups and the effects of treatment for data obtained from in vivo and in vitro studies were analyzed by one-way ANOVA, followed by the Tukey's test for multiple comparisons (GraphPad Prism version 7; GraphPad Prism Software, Inc.). $\mathrm{P}<0.05$ was considered to indicate a statistically significant difference.

\section{Results}

Effects of Dex on myocardial injuries in sepsis. H\&E staining showed changes in the hearts of the Ctrl, CLP, CLP + Dex and CLP + Dex + YOH mice (Fig. 1A). Moreover, cell nuclei appeared as blue dominant dots due to inflammation induced by CLP, and healthy cell nuclei were navy-blue in the control group, Dex treatment reduced the number of inflammatory cells, while $\mathrm{YOH}$ reversed the protective effects of Dex. TN-I is a classic marker of myocardial injury (29) and it was identified that CLP significantly increased TN-I (CLP vs. Ctrl, $\mathrm{P}<0.01$ ) (Fig. 1B), together with elevations in 8-OHDG and MDA (CLP vs. Ctrl, P<0.01) (Fig. 1C and D), which are markers of oxidative stress. Moreover, the antioxidant factor SOD (Fig. 1E) was significantly reduced in the CLP group compared with the $\mathrm{Ctrl}$ group $(\mathrm{P}<0.05)$. Following treatment with Dex, the CLP-induced increase in TN-I, MDA and 8-OHDG was significantly attenuated (CLP + Dex vs. CLP, $\mathrm{P}<0.05)$. Furthermore, SOD was significantly elevated following treatment with Dex (CLP + Dex vs. CLP, $\mathrm{P}<0.05)$. After co-treatment with $\mathrm{YOH}, 8-\mathrm{OHDG}$ levels were significantly increased and SOD decreased significantly (Fig. 1C and E); however, TN-I and MDA levels were not influenced by $\mathrm{YOH}(\mathrm{CLP}+\mathrm{Dex}+\mathrm{YOH}$ vs. CLP + Dex $)$.
Therefore, the present results indicated that the protective effects of Dex may be partly achieved by reducing oxidative stress.

Effects of Dex on reducing the expression of inflammatory factors. It was demonstrated that the expression levels of inflammatory factors IL-6 and MCP-1 were significantly increased in the CLP group compared with the $\mathrm{Ctrl}$ group $(\mathrm{P}<0.05$ and $\mathrm{P}<0.01$, respectively) (Fig. $2 \mathrm{~A}$ and $\mathrm{B}$ ). Furthermore, CLP significantly increased the protein expression levels of iNOS and P53 (CLP vs. Ctrl, P<0.01) (Fig. 2C and D). Following pre-treatment with Dex, the CLP-induced elevations of IL-6 and MCP-1 were significantly attenuated in the CLP + Dex group compared with the CLP group $(\mathrm{P}<0.05)$ (Fig. 2A and $\mathrm{B}$ ). In addition, Dex significantly decreased the protein expression levels of iNOS and P53 compared with CLP alone $(\mathrm{P}<0.05)$ (Fig. 2C and D). However, these protective effects of Dex were prevented by the $\alpha 2-\mathrm{AR}$ inhibitor $\mathrm{YOH}(\mathrm{CLP}+\mathrm{Dex}+\mathrm{YOH}$ vs. CLP + Dex, P<0.05).

Changes of ferroptosis with or without Dex. GPX4 is an indicator of and plays a central role in ferroptosis (13). The present results indicated that the protein expression of GPX4 and GSH release were significantly decreased by CLP (CLP vs. Ctrl, $\mathrm{P}<0.05$ ) (Fig. 3A and B), and that Dex significantly attenuated this decrease (CLP + Dex vs. CLP, $\mathrm{P}<0.05)$. After co-treatment with $\mathrm{YOH}$, Dex did not increase the protein expression of GPX4 or GSH level (CLP + Dex + YOH vs. CLP + Dex, P<0.01 and $\mathrm{P}<0.05$, respectively). It was found that following CLP, iron concentration was elevated from 8.1 to $15.66 \mu \mathrm{M}$ (CLP vs. Ctrl, $\mathrm{P}<0.01$ ) (Fig. 3C). Furthermore, protein expression levels of HO-1 (Fig. 3D), Nrf2 (Fig. 3E) and TFR (Fig. 3F and H) were increased (CLP vs. Ctrl, P<0.01), while that of ferritin, which accumulates iron (18), significantly decreased (CLP vs. Ctrl, $\mathrm{P}<0.05$ ) (Fig. 3G). Furthermore, Dex treatment significantly decreased iron concentration (from 15.66 to $9.7 \mu \mathrm{M}, \mathrm{P}<0.01$ ) (Fig. 3C) and the protein expression of HO-1 following CLP compared with CLP alone $(\mathrm{P}<0.05)$ (Fig. 3D). Dex treatment also moderately increased the protein expression of Nrf2, but the difference was not statistically significant (CLP + Dex vs. CLP). The expression of ferritin was significantly increased (Fig. 3G), while TFR expression was significantly decreased (Fig. $3 \mathrm{H}$ ) by Dex treatment (CLP + Dex vs. CLP, P<0.05). However, it was identified that $\mathrm{YOH}$ reversed these changes and led to myocardial injury (CLP + Dex + YOH vs. CLP + Dex, P<0.05) (Fig. 3).

Effects of Dex on apoptosis and pyroptosis during sepsis. Cleaved caspase 3, caspase 9, Bax and Bcl-2 are markers of apoptosis. The expression levels of cleaved caspase 3 and caspase 9 were both significantly increased by CLP (CLP vs. Ctrl, $\mathrm{P}<0.05$ ) (Fig. 4A and B). Moreover, it was found that the $\mathrm{Bax} / \mathrm{Bcl}-2$ ratio was significantly elevated (CLP vs. $\mathrm{Ctrl}, \mathrm{P}<0.01$ ) (Fig. 4C). It was identified that Dex treatment reduced the expression levels of cleaved caspase-3, caspase-9 and Bax/Bcl-2 to exert cardioprotective effects (CLP + Dex vs. CLP, $\mathrm{P}<0.05)$. However, YOH did not prevent the Dex-induced reduction in the expression levels of apoptotic proteins. Following treatment with YOH, cleaved caspase- 3 and caspase- 9 expression levels, and the $\mathrm{Bax} / \mathrm{Bcl}-2$ ratio were moderately elevated, but the difference was not significant. GSDMD plays a key role in pyroptosis (30) and it 

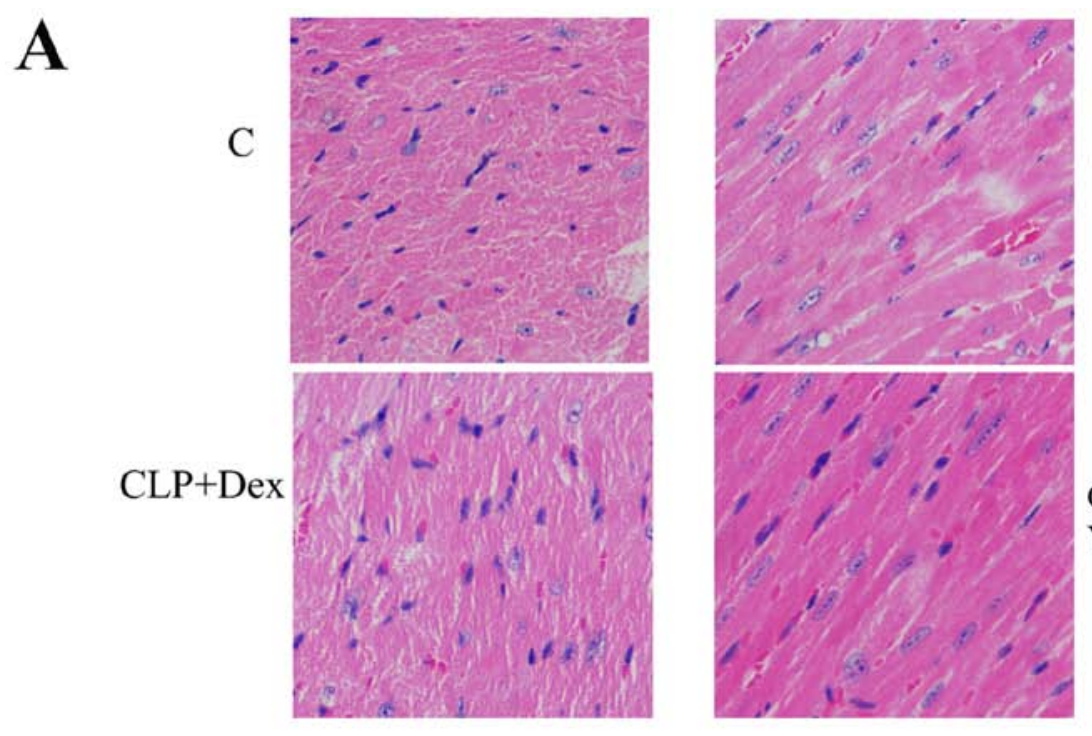

\section{CLP}
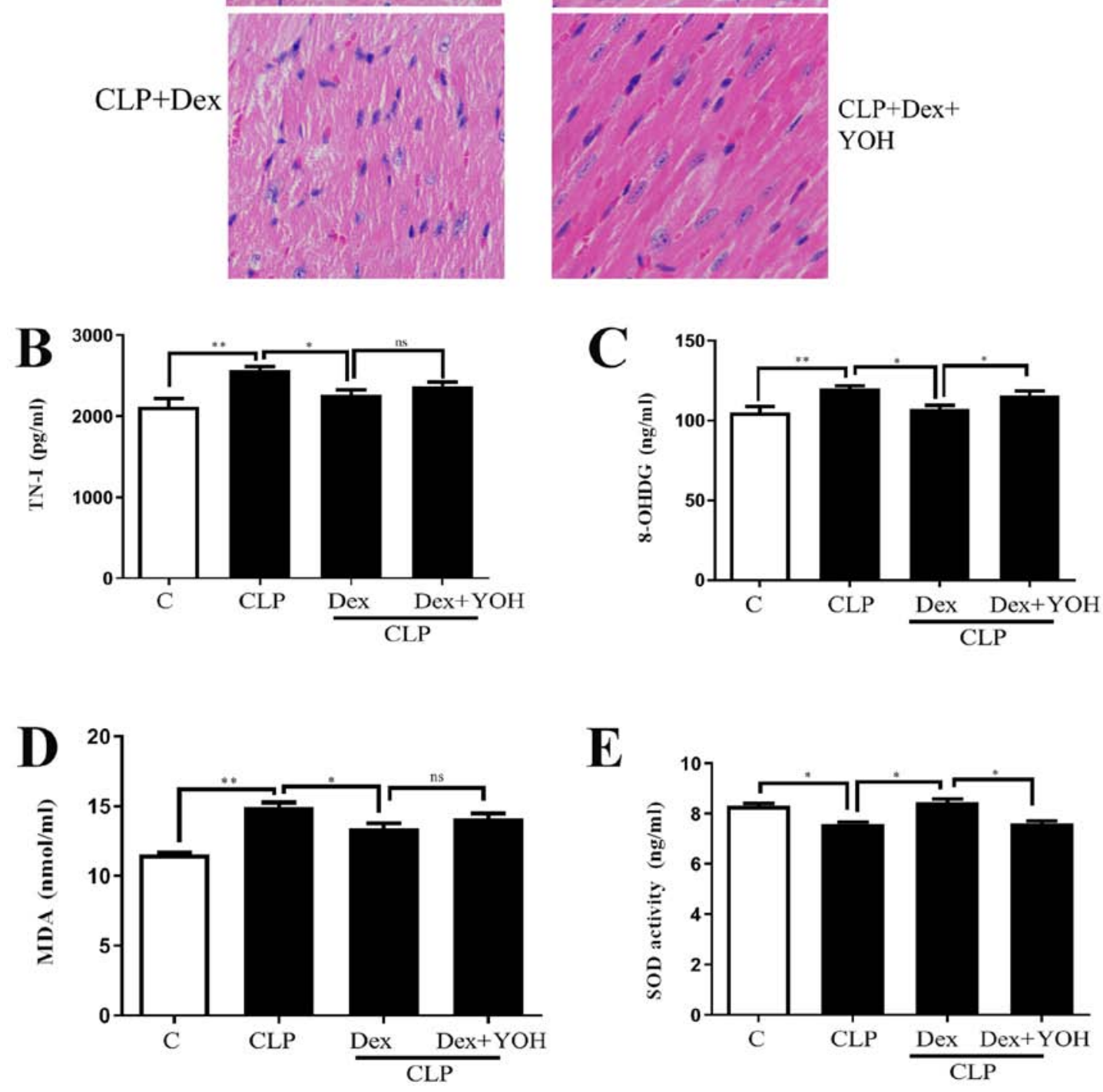

Figure 1. Effects of Dex on septic myocardial function. (A) Heart hematoxylin and eosin staining. Cell nuclei appeared as blue dominant dots due to CLP-induced inflammation, while normal cell nuclei were navy-blue. Dex treatment reduced inflammatory cells and YOH reversed the protective effects of Dex. Changes in (B) TN-I, (C) 8-OHDG, (D) SOD and (E) MDA release. Sepsis was achieved by CLP. Data are presented as the mean \pm SEM. $n=6$ per group. ${ }^{~} \mathrm{P}<0.05$ and ${ }^{* *} \mathrm{P}<0.01$. ns, not significant; Dex, dexmedetomidine; CLP, cecal ligation and puncture; YOH, yohimbine hydrochloride; TN-I, troponin-I; 8-OHDG, 8-hydroxy-2'-deoxyguanosine; SOD, superoxide dismutase; MDA, malonaldehyde; C, control.

was identified that CLP significantly increased the protein expression of GSDMD (CLP vs. Ctrl, P<0.01) (Fig. 4D). Furthermore, Dex treatment significantly reduced the CLP-induced increase in GSDMD (CLP + Dex vs. CLP, P<0.01), but YOH abolished this Dex-induced reduction of the protein expression of GSDMD $(\mathrm{CLP}+\mathrm{Dex}+\mathrm{YOH}$ vs. CLP + Dex, $\mathrm{P}<0.01)$.

\section{Discussion}

Sepsis causes whole body dysfunction due to infection and is one of the leading causes of mortality worldwide $(31,32)$. While it has been studied for $\sim 2,000$ years, the incidence of sepsis has not decreased over time (33). Inflammation is the first characteristic feature of sepsis. Previous studies have revealed that oxidative stress caused by inherent inflammatory responses can lead to the initiation of lipid peroxidation, DNA damage and mitochondrial function deterioration, as well as further the development of organ dysfunction and failure $(14,34)$. Therefore, increases in inflammation and oxidative stress both contribute to the damage caused by sepsis (32). Moreover, oxidative stress has been reported to participate in numerous pathological conditions, such as diabetes and myocardial 

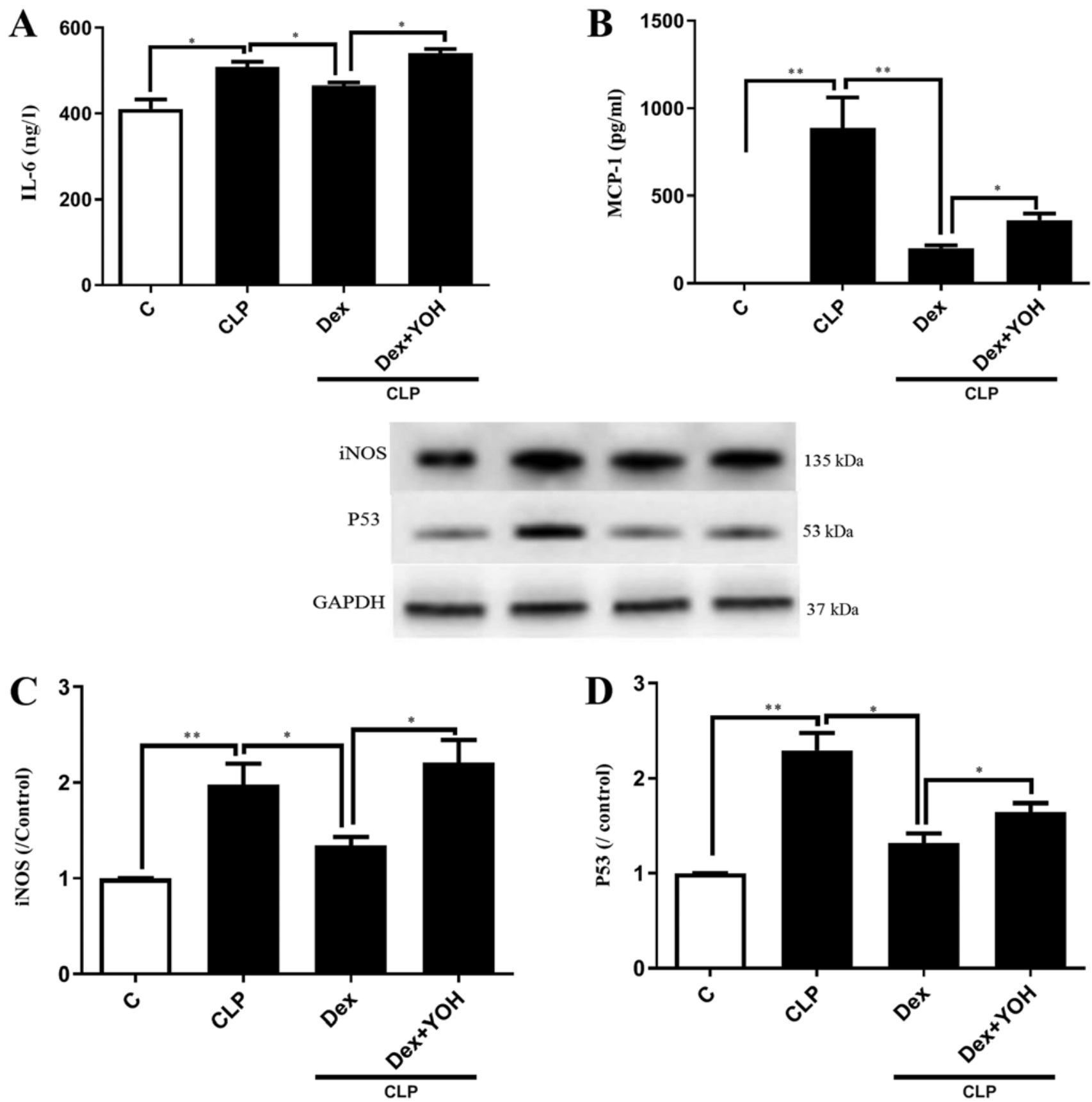

Figure 2. Effects of Dex on reducing the expression levels of inflammatory factors. Changes in (A) IL-6 and (B) MCP-1. Changes in the protein expression of (C) iNOS and (D) P53. Sepsis was achieved by CLP. Data are presented as the mean \pm SEM. $n=6$ per group. "P $<0.05$ and ${ }^{* *} \mathrm{P}<0.01$. Dex, dexmedetomidine; CLP, cecal ligation and puncture; YOH, yohimbine hydrochloride; IL-6, interleukin 6; MCP-1, monocyte chemoattractant protein-1; iNOS, nitric oxide synthase; $\mathrm{C}$, control.

ischemia reperfusion injury $(35,36)$. To maintain the balance of oxidants and antioxidants, an antioxidant defense system that involves GPX, SOD, catalase (37), ascorbic acid, GSH and $\alpha$-tocopherol plays crucial roles in decreasing oxidative stress levels. During sepsis, reactive nitrogen species, including the free radical NO and the non-radical peroxynitrite, was increased $(38,39)$. Dex, which exhibits superior anxiolytic and sedative effects without causing respiratory depression and with minor adverse effects, has been widely used in intensive care units and clinical anesthesia (40). Furthermore, Dex has been proven to exert protective effects against $\mathrm{H}_{2} \mathrm{O}_{2}$-induced myocardial cell injury by reducing oxidative stress; it can also reduce lung injury induced by lipopolysaccharide $(23,41)$ and confer other protective effects. In the present study, Dex was found to reduce CLP-induced heart injury by increasing SOD and GSH levels, and decreasing inflammatory factor levels, which was consistent with the findings of previous studies $(42,43)$.

Cell death, which mainly occurs via apoptosis, necrosis, pyroptosis and the recently discovered ferroptosis, not only kills cells, but can also play unique roles in various physiological and pathological conditions to maintain homeostasis (44). Apoptosis was recognized as the first type of programmed cell death as early as in 1960; its main characteristics include chromatin 


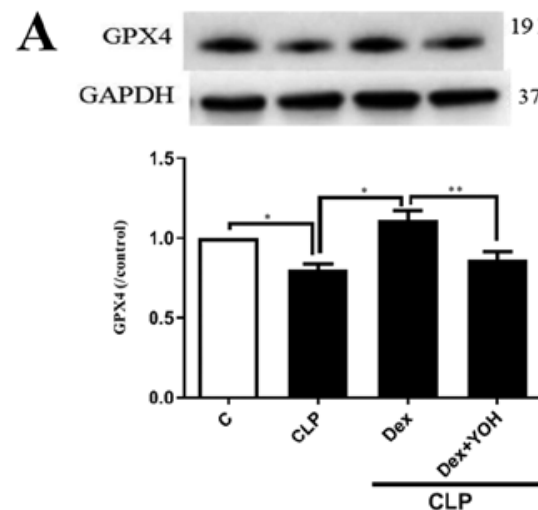

B

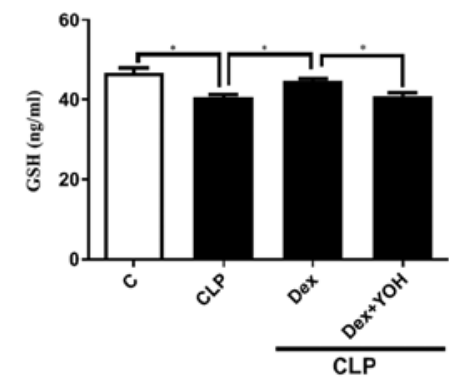

C

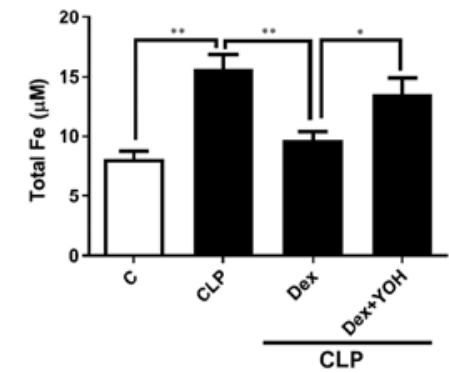

D
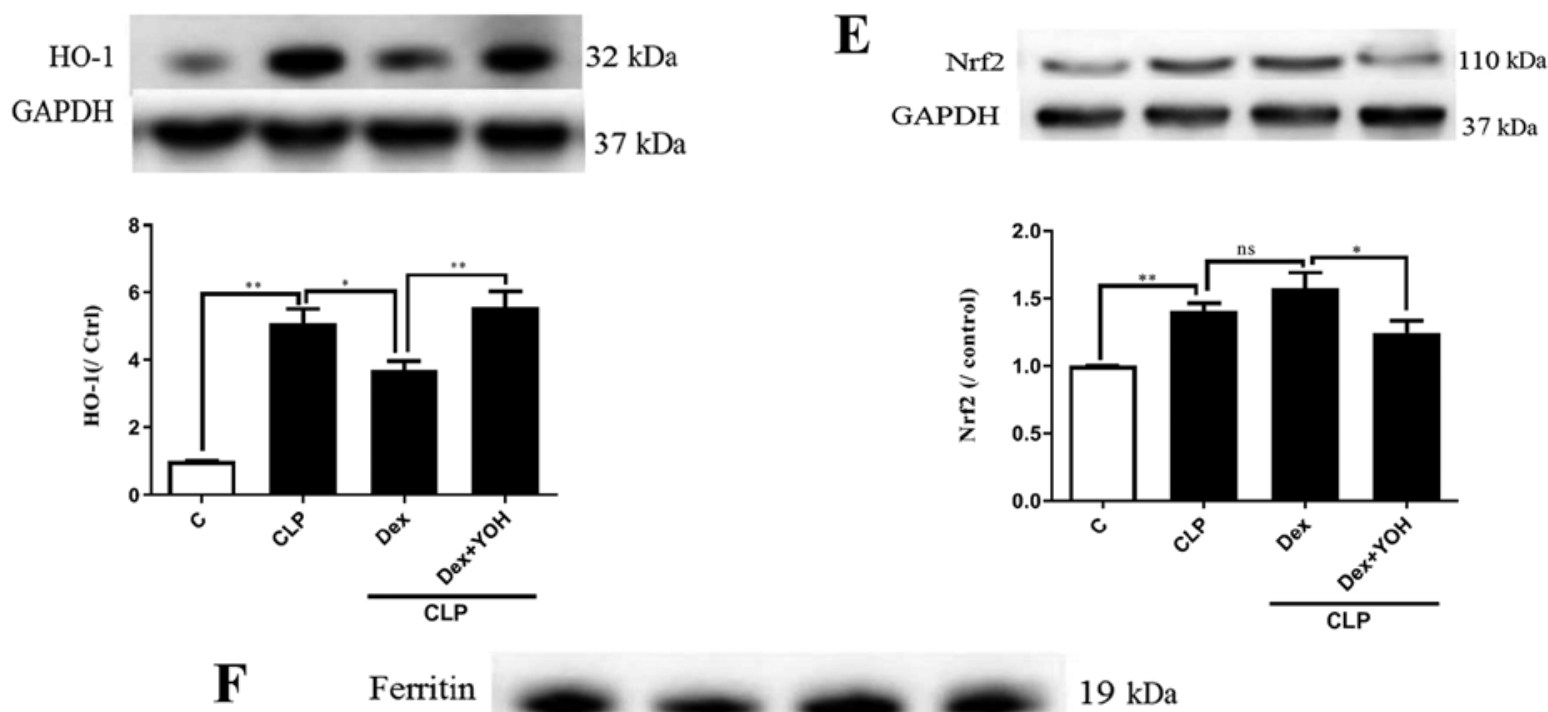

Ferritin

$19 \mathrm{kDa}$

TFR

GAPDH

$110 \mathrm{kDa}$

$37 \mathrm{kDa}$
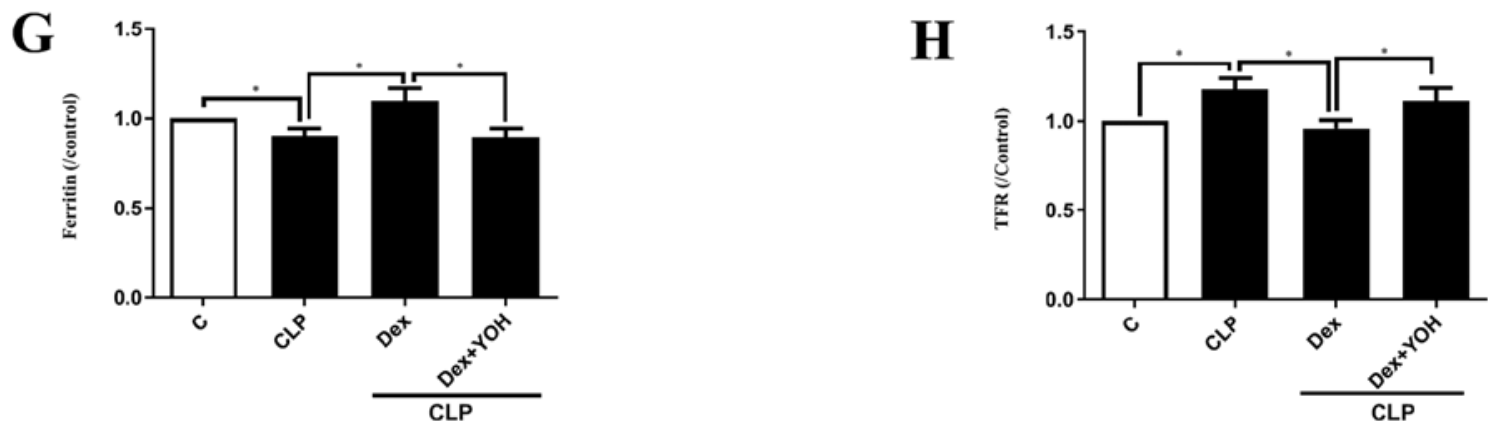

Figure 3. Changes in ferroptosis with or without Dex. (A) Changes in the protein expression of GPX4. (B) Changes in GSH release. (C) Changes in iron concentration. Changes in the protein expression levels of (D) HO-1 and (E) Nrf2. (F) Western blot band of (G) ferritin and (H) TFR. Sepsis was achieved by CLP. Data are presented as the mean \pm SEM. $n=6$ per group. ${ }^{*} \mathrm{P}<0.05$ and ${ }^{* *} \mathrm{P}<0.01$. Dex, dexmedetomidine; CLP, cecal ligation and puncture; $\mathrm{YOH}$, yohimbine hydrochloride; GPX4, glutathione peroxidase 4; GSH, glutathione; HO-1, heme oxygenase-1; Nrf2, nuclear factor E2-related factor 2; TFR, transferrin receptor; ns, normal control; C, control.

condensation and nuclear fragmentation, cell shrinkage, plasma membrane blebbing and apoptotic body formation without plasma membrane breakdown (45-47). In the beginning of the apoptotic process, caspases cleave and activate downstream factors, such as Bcl-2 and ROS, which can affect other types of cell death $(47,48)$. In addition to apoptosis, other types of regu- lated cell death, such as pyroptosis and ferroptosis, have also been shown to participate in different pathological processes (49). Pyroptosis is recognized as inflammasome-dependent cell death; during this process, the cell membrane loses its integrity, leading to the dissolution of the cell membrane and further induction of an inflammatory response. Activated NACHT-, LRR- and PYD 

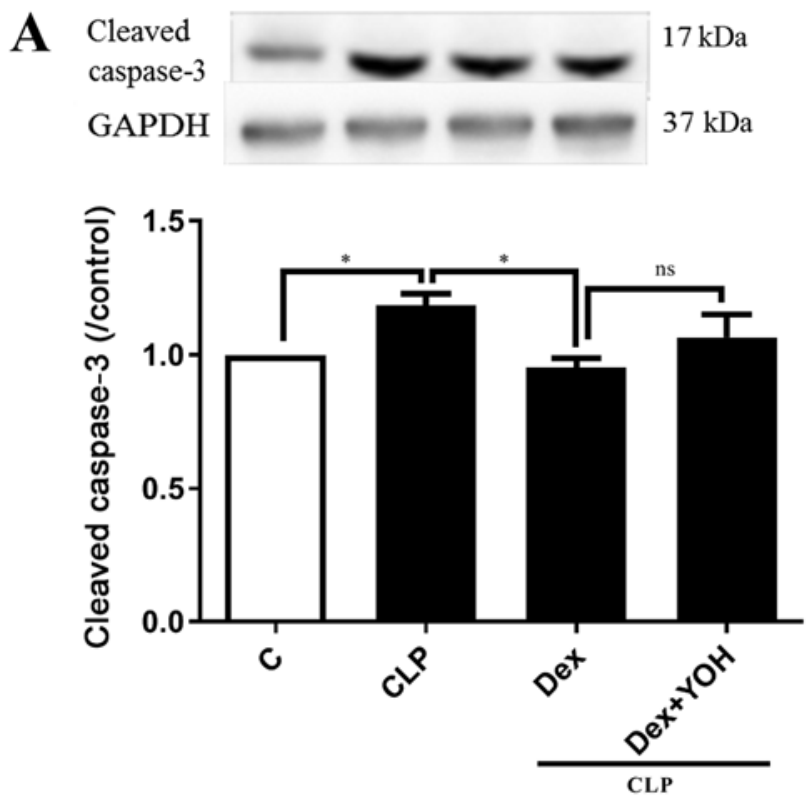

C
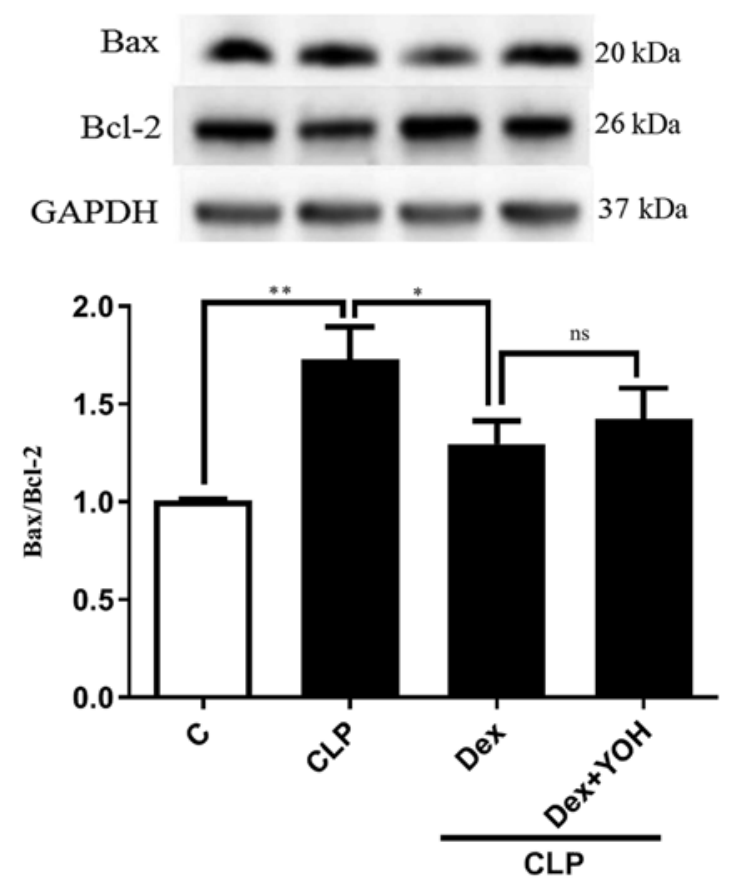
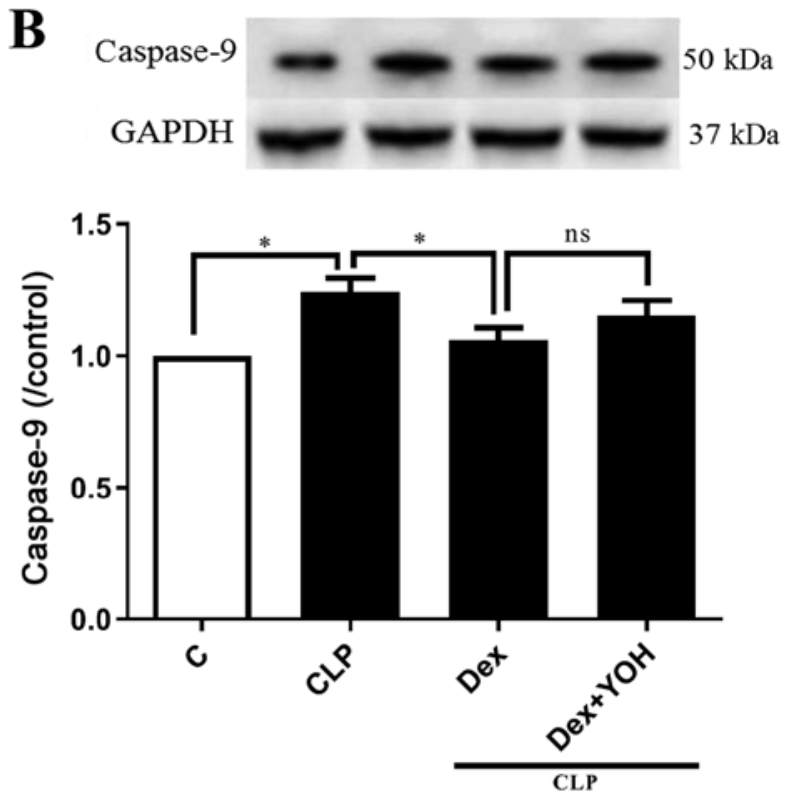

D
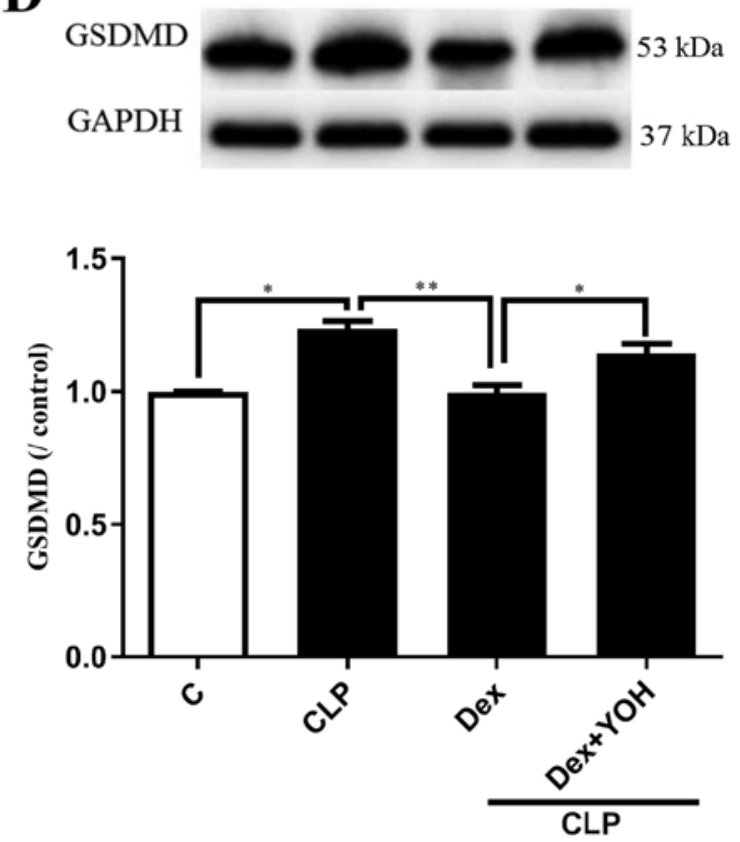

Figure 4. Effects of Dex on apoptosis and pyroptosis during sepsis. Changes in the protein expression levels of (A) cleaved caspase 3 and (B) caspase 9. (C) Bax/Bcl-2 ratio. (D) Changes in the protein expression of GSDMD. Sepsis was achieved by CLP. Data are presented as the mean \pm SEM. $\mathrm{n}=6$ per group. ${ }^{*} \mathrm{P}<0.05$ and ${ }^{* *} \mathrm{P}<0.01$. Dex, dexmedetomidine; GSDMD, gasdermin D; CLP, cecal ligation and puncture; YOH, yohimbine hydrochloride; ns, normal control; $\mathrm{C}$, control; ns, not significant.

domains-containing protein 3 (NALP3), a marker of pyroptosis, leads to the aggregation of cytokines IL- $1 \beta$ and caspase- 1 , which can be followed by tissue injury and organ dysfunction $(50,51)$. Moreover, inflammatory factors released during apoptosis and necroptosis lead to the induction of pyroptosis via inflammasome formation and caspase-1 activation, which, in turn, leads to the activation of GSDMD, another marker of pyroptosis (30). As previously reported, excessive ROS can activate NLR family pyrin domain containing 3 and lead to pyroptosis (52).

Ferroptosis is an iron-dependent form of cell death induced by erastin (15), and was recently shown to also be ROS-dependent. Furthermore, ferroptosis is different from apoptosis and pyroptosis. Antioxidants, such as $\mathrm{N}$-acetylcysteine and iron chelators, prevent ferroptosis, thus indicating that ferroptosis is a non-apoptotic but iron- and oxidative stress-dependent form of cell death (18). Ferroptosis mainly relies on regulators, such as GPX, HO-1, ferritin, transferrin, Nrf2, NADPH and voltage-dependent anion channel (12). Based on this, excessive ROS can lead to apoptosis, pyroptosis and ferroptosis, and once cell death has occurred, the subsequent release of inflammatory cytokines and overproduction of ROS plays a crucial role in inducing other types of cell death. Moreover, these types of cell death interact with each other (53). In the present study, it was found that during sepsis, GPX4 and GSH protein expression levels were decreased. In addition, it was demonstrated that Dex restored the expression levels of GPX4 and GSH, thus revealing 
that ferroptosis may be involved in sepsis. Furthermore, the expression of the antioxidant protein $\mathrm{HO}-1$ was increased in the septic group compared with the Ctrl group. The present results also indicated that, following treatment with Dex, the expression of HO-1 was decreased. The major function of HO-1 is to metabolize heme to produce carbon monoxide (4), biliverdin and iron, while it also exerts anticancer, anti-inflammatory, antiapoptotic, antiproliferative and antioxidant effects. However, it is unclear whether the sustained overexpression of HO-1 is beneficial, as HO-1 can regulate iron homeostasis (22).

Iron is crucial for oxygen transport, ATP production and DNA synthesis, which help maintain cellular function. However, excessive iron is detrimental to the redox balance and can further enhance the production of inflammatory factors, such as IL-1 $\beta$, IL- 6 and TNF- $\alpha$, leading to more damage (14). In the present study, iron concentration was found to be increased during sepsis and the iron-related proteins TFR and ferritin were increased and decreased, respectively. It was speculated that these effects may possibly be due to the overexpression of HO-1, further demonstrating that ferroptosis participates in sepsis, but the definite relationship between iron and HO-1 requires further research. To date, previous studies have focused on the beneficial effects of HO-1, but have ignored the iron-downstream of HO-1, which may be toxic. Thus, the effects of a sustained increase in HO-1 expression under pathological conditions require further study. Previous studies have demonstrated that HO-1 regulates ferroptosis via iron, thus leading to septic heart injury $(54,55)$. The present results suggested that Dex reduced CLP-induced overexpression of HO-1 and increased the expression levels of GPX4 and Nrf2 to exert a protective effect, which may contradict previous findings that HO-1 is further enhanced following antioxidant treatment (56). One of the reasons for these differences could because different models were used in each study. Additionally, the primary focus in the present study was the relationship between HO-1 and ferroptosis, whereas their study focused on the role of HO-1 in antioxidant treatment. Therefore, future studies should further explore the role of HO-1. Hu et al (57) showed that Dex alleviates iron overload-induced injury in SH-SY5Y cells via antioxidative, anti-inflammatory and antiapoptotic mechanisms. Therefore, these findings provided evidence that iron overload is detrimental and that the effects of Dex are associated with iron. In the present study, it was speculated that Dex may exert its protective effects partly by reducing the expression of HO-1, thus also decreasing iron overload.

Moreover, YOH, an $\alpha 2-\mathrm{AR}$ antagonist, was used in the present study and was found to reverse the effects of Dex on HO-1 and GPX4. Therefore, Dex may exert its protective role mainly via $\alpha 2-A R$ to reduce ferroptosis. However, YOH did not prevent Dex from reducing the expression of the apoptotic protein cleaved caspase 3 or the $\mathrm{Bax} / \mathrm{Bcl}-2$ ratio. This result was consistent with the results of our preliminary study, in which $\mathrm{YOH}$ also did not affect $\mathrm{H}_{2} \mathrm{O}_{2}$ induced apoptosis. Therefore, the present results indicated that Dex exerted its cardioprotective effects partly by decreasing apoptosis in an $\alpha 2$-AR-independent manner. The primary aim of the present study was to investigate the effects of Dex on sepsis-induced ferroptosis, while the effectiveness of Dex in relation to oxidative stress among others was used as supporting data for comparison with previous studies. Thus, the present study only assessed the representative oxidative related parameters 8-OHDG, SOD and MDA. Therefore, further studies are required to examine other oxidative stress parameters, such as 4-HNE, catalase and NO estimation for a complete understanding of the effects of Dex on oxidative stress.

Sepsis causes significant adverse effects on both the cardiac myocytes and the myocardial vasculature, following its effects on systemic inflammation. The present study was a pilot study investigating the effects of Dex on sepsis-induced myocardial injury. In relation to ferroptosis, future studies should focus on the effects of Dex on sepsis-induced changes to the vasculature, including adhesion molecules, such as intercellular adhesion molecule-1. Dex is a selective $\alpha 2-\mathrm{AR}$ and was also previously proven to be an imidazoline receptor (58). Moreover, YOH is a potent $\alpha 2$-AR antagonist, which was used in the present study. Furthermore, further studies assessing Dex will use the agonist of $\alpha 2$-AR to clarify the effects of Dex and to identify the role of imidazoline receptor, which may facilitate the understanding of the underlying mechanism of Dex.

In conclusion, it was found that HO-1 regulated ferroptosis by regulating iron concentrations. Moreover, Dex exerted cardioprotective effects via ferroptosis reduction, by reducing iron concentration, the protein expression of HO-1 and inflammatory factors, as well as increasing the expression of GPX4. To the best of our knowledge, the present study is the first to focus on the association between Dex and ferroptosis in septic heart injury, and these results may provide further insights into the mechanism of the protective effects of Dex.

\section{Acknowledgements}

Not applicable.

\section{Funding}

This study was supported by funding from the National Natural Science Foundation of China (grant no. 81801947), Shenzhen Science and Technology Innovation Committee (grant no. JCYJ20180305180809671) and the Cooperative Research and Development Program of Shenzhen People's Hospital (grant no. SYJY201709).

\section{Availability of data and materials}

All data generated or analyzed during this study are included in this published article.

\section{Authors' contributions}

CW, YL and ZZ designed the experiments. CW, WY, AH and JL performed the experiments. CW, WY and AH analyzed the data. CFY and ZX contributed to data interpretation and manuscript revision. $\mathrm{CW}$ wrote the paper.

\section{Ethics approval and consent to participate}

This study was approved by the Institutional Ethics Committee of: The Medical Committee of Shenzhen People's Hospital (approval ID: LL-KY-2019599). 


\section{Patient consent for publication}

Not applicable.

\section{Competing interests}

The authors declare that they have no competing interests.

\section{References}

1. Sun X, Dai Y, Tan G, Liu Y and Li N: Integration analysis of $\mathrm{m}(6) \mathrm{A}-\mathrm{SNPs}$ and eQTLs associated with sepsis reveals platelet degranulation and staphylococcus aureus infection are mediated by m(6)A mRNA methylation. Front Genet 11: 7, 2020.

2. Parrillo JE: The cardiovascular pathophysiology of sepsis. Annu Rev Med 40: 469-485, 1989.

3. Ernsberger P, Giuliano R, Willette RN and Reis DJ: Role of imidazole receptors in the vasodepressor response to clonidine analogs in the rostral ventrolateral medulla. J Pharmacol Exp Ther 253: 408-418, 1990.

4. Barr J, Fraser GL, Puntillo K, Ely EW, Gélinas C, Dasta JF, Davidson JE, Devlin JW, Kress JP, Joffe AM, et al: Clinical practice guidelines for the management of pain, agitation, and delirium in adult patients in the intensive care unit. Crit Care Med 41: 263-306, 2013.

5. Taniguchi T, Kidani Y, Kanakura $\mathrm{H}$, Takemoto $\mathrm{Y}$ and Yamamoto K: Effects of dexmedetomidine on mortality rate and inflammatory responses to endotoxin-induced shock in rats. Crit Care Med 32: 1322-1326, 2004.

6. Venn RM, Bradshaw CJ, Spencer R, Brealey D, Caudwell E, Naughton C, Vedio A, Singer M, Feneck R, Treacher D, et al: Preliminary UK experience of dexmedetomidine, a novel agent for postoperative sedation in the intensive care unit Anaesthesia 54: 1136-1142, 1999.

7. Kong W, Kang K, Gao Y, Liu H, Meng X, Yang S, Yu K and Zhao M: Dexmedetomidine alleviates LPS-induced septic cardiomyopathy via the cholinergic anti-inflammatory pathway in mice. Am J Transl Res 9: 5040-5047, 2017.

8. Ji F, Li Z, Nguyen H, Young N, Shi P, Fleming $\mathrm{N}$ and Liu $\mathrm{H}$ : Perioperative dexmedetomidine improves outcomes of cardiac surgery. Circulation 127: 1576-1584, 2013.

9. Dolma S, Lessnick SL, Hahn WC and Stockwell BR: Identification of genotype-selective antitumor agents using synthetic lethal chemical screening in engineered human tumor cells. Cancer Cell 3: 285-296, 2003

10. Fang X, Wang H, Han D, Xie E, Yang X, Wei J, Gu S, Gao F, Zhu N, Yin X, et al: Ferroptosis as a target for protection against cardiomyopathy. Proc Natl Acad Sci USA 116: 2672-2680, 2019.

11. Gao M, Monian P, Quadri N, Ramasamy R and Jiang X: Glutaminolysis and transferrin regulate ferroptosis. Mol Cell 59: 298-308, 2015

12. Xie Y, Hou W, Song X, Yu Y, Huang J, Sun X, Kang R and Tang D: Ferroptosis: Process and function. Cell Death Differ 23: 369-379, 2016.

13. Zhu H, Santo A, Jia Z and Robert Li Y: GPx4 in bacterial infection and polymicrobial sepsis: Involvement of ferroptosis and pyroptosis. React Oxyg Species (Apex) 7: 154-160, 2019

14. Zhou B, Zhang JY, Liu XS, Chen HZ, Ai YL, Cheng K, Sun RY, Zhou D, Han J and Wu Q: Tom 20 senses iron-activated ROS signaling to promote melanoma cell pyroptosis. Cell Res 28: $1171-1185,2018$

15. Dixon SJ, Lemberg KM, Lamprecht MR, Skouta R, Zaitsev EM, Gleason CE, Patel DN, Bauer AJ, Cantley AM, Yang WS, et al: Ferroptosis: An iron-dependent form of nonapoptotic cell death. Cell 149: 1060-1072, 2012

16. Dixon SJ and Stockwell BR: The role of iron and reactive oxygen species in cell death. Nat Chem Biol 10: 9-17, 2014.

17. Baba Y, Higa JK, Shimada BK, Horiuchi KM, Suhara T, Kobayashi M, Woo JD, Aoyagi H, Marh KS, Kitaoka H and Matsui T: Protective effects of the mechanistic target of rapamycin against excess iron and ferroptosis in cardiomyocytes. Am J Physiol Heart Circ Physiol 314: H659-H668, 2018.

18. Zhou B, Liu J, Kang R, Klionsky DJ, Kroemer G and Tang D: Ferroptosis is a type of autophagy-dependent cell death. Semin Cancer Biol: Mar 14, 2019 doi: 10.1016/j.semcancer.2019.03.002 (Epub ahead of print).
19. Jin X, Xu Z, Cao J, Yan R, Xu R, Ran R, Ma Y, Cai W, Fan R, Zhang Y, et al: HO-1/EBP interaction alleviates cholesterol-induced hypoxia through the activation of the AKT and Nrf2/mTOR pathways and inhibition of carbohydrate metabolism in cardiomyocytes. Int J Mol Med 39: 1409-1420, 2017.

20. Andreas M, Oeser C, Kainz FM, Shabanian S, Aref T, Bilban M, Messner B, Heidtmann J, Laufer G, Kocher A and Wolzt M: Intravenous heme arginate induces HO-1 (Heme Oxygenase-1) in the human heart. Arterioscler Thromb Vasc Biol 38: 2755-2762, 2018.

21. Kwon MY, Park E, Lee SJ and Chung SW: Heme oxygenase-1 accelerates erastin-induced ferroptotic cell death. Oncotarget 6 : 24393-24403, 2015.

22. NaveenKumar SK, Hemshekhar M, Kemparaju K and Girish KS: Hemin-induced platelet activation and ferroptosis is mediated through ROS-driven proteasomal activity and inflammasome activation: Protection by melatonin. Biochim Biophys Acta Mol Basis Dis 1865: 2303-2316, 2019

23. Liu XR, Li T, Cao L, Yu YY, Chen LL, Fan XH, Yang BB and Tan XQ: Dexmedetomidine attenuates $\mathrm{H}_{2} \mathrm{O}_{2}$-induced neonatal rat cardiomyocytes apoptosis through mitochondria- and ER-medicated oxidative stress pathways. Mol Med Rep 17: 7258-7264, 2018

24. Qiu R, Yao W, Ji H, Yuan D, Gao X, Sha W, Wang F, Huang P and Hei Z: Dexmedetomidine restores septic renal function via promoting inflammation resolution in a rat sepsis model. Life Sci 204: 1-8, 2018

25. Xu L, Bao H, Si Y and Wang X: Effects of dexmedetomidine on early and late cytokines during polymicrobial sepsis in mice. Inflamm Res 62: 507-514, 2013.

26. Chen JH, Yu GF, Jin SY, Zhang WH, Lei DX, Zhou SL and Song XR: Activation of alpha2 adrenoceptor attenuates lipopolysaccharide-induced hepatic injury. Int J Clin Exp Pathol 8: 10752-10759, 2015

27. Zhao W, Jia L, Yang HJ, Xue X, Xu WX, Cai JQ, Guo RJ and Cao CC: Taurine enhances the protective effect of Dexmedetomidine on sepsis-induced acute lung injury via balancing the immunological system. Biomed Pharmacother 103: 1362-1368, 2018

28. Rinaldi B, Di Filippo C, Capuano A, Donniacuo M, Sodano L, Ferraraccio F, Rossi F and D'Amico M: Adiponectin elevation by telmisartan ameliorates ischaemic myocardium in Zucker diabetic fatty rats with metabolic syndrome. Diabetes Obes Metab 14: 320-328, 2012

29. Szekely L, Vijay P, Sharp TG, Bando K and Brown JW: Correlation of plasma adrenomedullin to myocardial preservation during open-heart surgery. Pediatr Cardiol 21: 228-233, 2000.

30. Shi J, Gao W and Shao F: Pyroptosis: Gasdermin-mediated programmed necrotic cell death. Trends Biochem Sci 42: 245-254, 2017.

31. Mantzarlis K, Tsolaki V and Zakynthinos E: Role of oxidative stress and mitochondrial dysfunction in sepsis and potential therapies. Oxid Med Cell Longev 2017: 5985209, 2017.

32. Lee WJ, Chen YL, Chu YW and Chien DS: Comparison of glutathione peroxidase-3 protein expression and enzyme bioactivity in normal subjects and patients with sepsis. Clin Chim Acta 489: 177-182, 2019.

33. Wang Y, Mao X, Chen H, Feng J, Yan M, Wang Y and Yu Y: Dexmedetomidine alleviates LPS-induced apoptosis and inflammation in macrophages by eliminating damaged mitochondria via PINK1 mediated mitophagy. Int Immunopharmacol 73: 471-481, 2019.

34. Kaludercic N and Di Lisa F: Mitochondrial ROS formation in the pathogenesis of diabetic cardiomyopathy. Front Cardiovasc Med 7: 12, 2020

35. Ighodaro OM: Molecular pathways associated with oxidative stress in diabetes mellitus. Biomed Pharmacother 108: 656-662, 2018.

36. Jin JK, Blackwood EA, Azizi K, Thuerauf DJ, Fahem AG, Hofmann C, Kaufman RJ, Doroudgar S and Glembotski CC: ATF6 decreases myocardial ischemia/reperfusion damage and links ER stress and oxidative stress signaling pathways in the heart. Circ Res 120: 862-875, 2017.

37. Catalao CHR, Santos-Júnior NN, da Costa LHA, Souza AO, Alberici LC and Rocha MJA: Brain oxidative stress during experimental sepsis is attenuated by simvastatin administration. Mol Neurobiol 54: 7008-7018, 2017.

38. Droge W: Free radicals in the physiological control of cell function. Physiol Rev 82: 47-95, 2002 
39. Webster NR and Nunn JF: Molecular structure of free radicals and their importance in biological reactions. Br J Anaesth 60: 98-108, 1988

40. Arcangeli A, D'Alò C and Gaspari R: Dexmedetomidine use in general anaesthesia. Curr Drug Targets 10: 687-695, 2009.

41. Fu C, Dai X, Yang Y, Lin M, Cai Y and Cai S: Dexmedetomidine attenuates lipopolysaccharide-induced acute lung injury by inhibiting oxidative stress, mitochondrial dysfunction and apoptosis in rats. Mol Med Rep 15: 131-138, 2017.

42. Sha J, Zhang H, Zhao Y, Feng X, Hu X, Wang C, Song M and Fan H: Dexmedetomidine attenuates lipopolysaccharide-induced liver oxidative stress and cell apoptosis in rats by increasing GSK-3 $\beta /$ MKP-1/Nrf2 pathway activity via the $\alpha 2$ adrenergic receptor. Toxicol Appl Pharmacol 364: 144-152, 2019.

43. Yazar E, Er A, Uney K, Bulbul A, Avci GE, Elmas M and Tras B Effects of drugs used in endotoxic shock on oxidative stress and organ damage markers. Free Radic Res 44: 397-402, 2010.

44. Fearnhead HO, Vandenabeele P and Vanden Berghe T: How do we fit ferroptosis in the family of regulated cell death? Cell Death Differ 24: 1991-1998, 2017.

45. Zhu $\mathrm{H}$ and Sun A: Programmed necrosis in heart disease: Molecular mechanisms and clinical implications. J Mol Cell Cardiol 116: 125-134, 2018.

46. Mughal W, Dhingra R and Kirshenbaum LA: Striking a balance: Autophagy, apoptosis, and necrosis in a normal and failing heart. Curr Hypertens Rep 14: 540-547, 2012.

47. Konstantinidis K, Whelan RS and Kitsis RN: Mechanisms of cell death in heart disease. Arterioscler Thromb Vasc Biol 32: $1552-1562,2012$

48. Galluzzi L, Kepp O, Krautwald S, Kroemer G and Linkermann A: Molecular mechanisms of regulated necrosis. Semin Cell Dev Biol 35: 24-32, 2014.

49. Li Z, Jia Y, Feng Y, Cui R, Miao R, Zhang X, Qu K, Liu C and Zhang J: Methane alleviates sepsis-induced injury by inhibiting pyroptosis and apoptosis: In vivo and in vitro experiments. Aging (Albany NY) 11: 1226-1239, 2019.

50. Fu Q, Wu J, Zhou XY, Ji MH, Mao QH, Li Q, Zong MM, Zhou ZQ and Yang JJ: NLRP3/caspase-1 pathway-induced pyroptosis mediated cognitive deficits in a mouse model of sepsis-associated encephalopathy. Inflammation 42: 306-318, 2019.
51. Patel S: Inflammasomes, the cardinal pathology mediators are activated by pathogens, allergens and mutagens: A critical review with focus on NLRP3. Biomed Pharmacother 92: 819-825, 2017.

52. Zhou W, Chen C, Chen Z, Liu L, Jiang J, Wu Z, Zhao M and Chen Y: NLRP3: A novel mediator in cardiovascular disease. J Immunol Res 2018: 5702103, 2018.

53. Bruni A, Bornstein S, Linkermann A and Shapiro AMJ: Regulated cell death seen through the lens of islet transplantation. Cell Transplant 27: 890-901, 2018

54. Li C, Lönn ME, Xu X, Maghzal GJ, Frazer DM, Thomas SR, Halliwell B, Richardson DR, Anderson GJ and Stocker R: Sustained expression of heme oxygenase-1 alters iron homeostasis in nonerythroid cells. Free Radic Biol Med 53: 366-374, 2012.

55. Soares MP, Seldon MP, Gregoire IP, Vassilevskaia T, Berberat PO, Yu J, Tsui TY and Bach FH: Heme oxygenase-1 modulates the expression of adhesion molecules associated with endothelial cell activation. J Immunol 172: 3553-3563, 2004

56. Mao X, Wang T, Liu Y, Irwin MG, Ou JS, Liao XL, Gao X, $\mathrm{Xu}$ Y, Ng KF, Vanhoutte PM and Xia Z: N-acetylcysteine and allopurinol confer synergy in attenuating myocardial ischemia injury via restoring $\mathrm{HIF}-1 \alpha / \mathrm{HO}-1$ signaling in diabetic rats. PLoS One 8: e68949, 2013.

57. Hu XB, Xi ZY, Liu LQ, Kang K, Li WH, Shen YX, Kang F and Li J: Dexmedetomidine promotes SH-SY5Y cell resistance against impairment of iron overload by inhibiting NF- $\mathrm{B}$ Pathways. Neurochem Res 44: 959-967, 2019.

58. Zhang F, Ding T, Yu L, Zhong Y, Dai $\mathrm{H}$ and Yan $\mathrm{M}$ : Dexmedetomidine protects against oxygen-glucose deprivation-induced injury through the I2 imidazoline receptor-PI3K/AKT pathway in rat C6 glioma cells. J Pharm Pharmacol 64: 120-127, 2012.

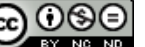

This work is licensed under a Creative Commons Attribution-NonCommercial-NoDerivatives 4.0 International (CC BY-NC-ND 4.0) License. 